\title{
DYNAMIC STIFFNESS MATRICES FOR TWO CIRCULAR FOUNDATIONS
}

\author{
GIN-SHOW LIOU \\ Department of Civil Engineering. National Chiao-Tung University, 1001 Ta Hsueh Road, Hsin-Chu 30049, Taiwan
}

\begin{abstract}
SUMMARY
A systematic procedure is presented for generating dynamic stiffness matrices for two independent circular foundations on an elastic half-space medium. With the technique reported in References $1-3$, the analytic solution of threedimensional (3D) wave equations satisfying the prescribed traction due to the vibration of one circular foundation can be found. Since there are two analytic solutions for two prescribed tractions due to the vibrations of two circular foundations, the principle of superposition must be used to obtain the total solution. The interaction stresses (prescribed tractions) are assumed to be piecewise linear in the $r$-directions of both cylindrical co-ordinates for the two circular foundations. Then, the variational principle and the reciprocal theorem are employed to generate the dynamic stiffness matrices for the two foundations. In the process of employing the variational principle, a co-ordinate transformation matrix between two cylindrical co-ordinate systems is introduced. Some numerical results of dynamic stiffness matrices for the interaction of two identical rigid circular foundations are presented in order to show the effectiveness and efficiency of the present method, and some elaborations for its future extensions are also discussed.
\end{abstract}

\section{INTRODUCTION}

Soil-structure interaction problems have attracted much attention from researchers in earthquake engineering in the past few decades. To perform soil-structure interaction analysis, a sub-structure technique is often used. In sub-structuring analysis, the interaction of the structure and the surrounding soil medium is usually represented by a dynamic stiffness matrix, which can be combined with the total stiffness matrix of the finite element model of the structure.

To generate the dynamic stiffness of a structural foundation, some analytic procedures are available. For example, Lysmer ${ }^{4}$ employed the analytical solution for constant normal ring-traction on a half-space medium to generate the compliance function (the inverse of the dynamic stiffness function) for vertical vibration of a rigid circular plate, Luco and Westmann ${ }^{5}$ obtained the compliance functions for torsional, vertical, horizontal and rocking vibrations of a rigid circular plate by reducing the Fredholm integral equations to algebraic equations using the finite difference method, and Wong and Luco ${ }^{6}$ employed an idea similar to Lysmer's to generate the compliance functions for a rigid foundation with an arbitrary shape on a half-space medium. Liou $^{1-3}$ developed a technique to generate dynamic stiffness functions for vibrations of an axial symmetric foundation and a foundation with an arbitrary shape.

When two independent foundations are sufficiently close, their interaction is significant in the seismic analysis of the two structures. Therefore, researchers have started to pay attention to structure-soil-structure interaction in recent decades. Similarly, to perform structure-soil-structure interaction analysis, a substructure technique can also be employed. Thus, to generate the dynamic stiffness matrix for a twofoundation system is an important step in the analysis of structure-soil-structure interaction.

To generate the dynamic stiffness functions, many procedures are available. For example, Roesset and Gonzalez ${ }^{7}$ employed the semi-analytical solution of unit loading on a layered stratum, which is obtained by using the consistent boundary method (layer element) to generate the dynamic stiffness functions réquired and then investigating the behaviour of the interaction of the two structures. Lin et al. ${ }^{8}$ also used a hybrid method in which the near field and the complementary far field of a layered stratum are modelled by the finite 
element method and the consistent boundary method, respectively, to investigate the behaviour of the vibrations of two embedded foundations. Warburton et al. ${ }^{9}$ employed the solution for a single foundation to approximate the displacement at the location of the second foundation by an averaging process. This method can give fair results for low-frequency excitation. Savidis and Richter ${ }^{10}$ used Green's function to generate the boundary integral equation for a two-foundation system. For two-dimensional (2-D) problems, Luco and Contesse ${ }^{11}$ obtained an analytic form of the solution for two shear walls subjected to anti-plane shear wave (SH waves) cxcitations, and studied the interaction effect of these two walls. Murakami and Luco ${ }^{12}$ extended the solution to investigate the interaction behaviour among many shear walls which are equally spaced. For 3-D problems, Wong and Luco ${ }^{13}$ used a procedure similar to that of Savidis to obtain the boundary integral equation for a multiple foundation system. They also developed a technique for an iterative approach, which can save some computational effort when finding the inverse of the complex matrix of the influence function. Kawakami and Tasaki ${ }^{14}$ directly employed the boundary element method and developed a simplified method to study the interaction effect of two masses excited by wave motion in a half-space. Triantafyllidis and Prange ${ }^{15-16}$ employed the influence function, which is the integral of Green's function, to generate the dynamic stiffness functions for two rigid circular foundations and two rigid rectangular foundations.

This paper presents a systematic procedure to generate the dynamic stiffness matrix for two circular foundations on a half-space medium. In the procedure, a technique, reported in References 1 3, of decomposing arbitrarily prescribed stress boundary conditions on the surface of a half-space medium is used to find the analytical solutions of 3-D wave equations in cylindrical co-ordinates. The interaction stresses of the foundations and the soil medium are assumed to be piecewise linear in the $r$-direction. Two solutions for the interaction stresses from the vibrations of the two foundations are obtained separately, and then the principle of superposition is used to obtain the final solution. After that the variational principle is employed to generate the dynamic stiffness matrix for the two foundations. In the process of using the variational principle, the co-ordinate transformation matrix is introduced, since the two independent solutions are described in different cylindrical co-ordinate systems.

Some numerical results are presented for the dynamic stiffness matrices for two identical rigid circular foundations, with different distances apart, on a half-space medium. A comparison is made with the results in Reference 15 in order to show the effectiveness and efficiency of the present method and shed some light on the significance of the effect of structure-soil-structure interaction. Some comments on the important features of this method are also presented.

\section{FORMULATION OF A DYNAMIC STIFFNESS MATRIX}

To generate a dynamic stiffness matrix for two circular foundations, a sub-structure technique is employed. For the sub-structure of wave propagation in a half-space medium, the contact stresses (interaction stresses) due to the harmonic vibration of the foundation are treated as the prescribed tractions on the surface of a half-space medium, as shown in Figure 1. In Figure 1, the prescribed traction $\mathbf{t}_{1}$ (in contact area No. 1), described in an $r_{1}, \theta_{1}, z_{1}$ co-ordinate system, and the prescribed traction $t_{2}$ (in contact area No. 2), described in an $r_{2}, \theta_{2}, z_{2}$ co-ordinate system, can be expressed in terms of Fourier components with respect to $\theta_{1}$ and $\theta_{2}$ in the following two equations, respectively.

and

$$
\mathbf{t}_{1}=\left\{\begin{array}{l}
\bar{\tau}_{r_{1} z_{1}}\left(r_{1}, \theta_{1}\right) \\
\bar{\sigma}_{z_{1} z_{1}}\left(r_{1}, \theta_{1}\right) \\
\bar{\tau}_{\theta_{1} z_{1}}\left(r_{1}, \theta_{1}\right)
\end{array}\right\}=\sum_{n=0}^{\infty} \mathbf{L}_{n}^{\mathrm{s}}\left\{\begin{array}{l}
\bar{\tau}_{r_{1} z_{1}, n}^{\mathrm{s}}\left(r_{1}\right) \\
\bar{\sigma}_{z_{2}}^{\mathrm{s}} z_{1, n}\left(r_{1}\right) \\
\bar{\tau}_{\theta_{1} z_{1}, n}^{\mathrm{s}}\left(r_{1}\right)
\end{array}\right\}+\mathbf{L}_{n}^{\mathrm{a}}\left\{\begin{array}{l}
\bar{\tau}_{\mathrm{r}_{1} z_{1}, n}^{\mathrm{a}}\left(r_{1}\right) \\
\bar{\sigma}_{z_{1} z_{1}, n}^{\mathrm{a}}\left(r_{1}\right) \\
\bar{\tau}_{\theta_{1} z_{1}, n}^{\mathrm{a}}\left(r_{1}\right)
\end{array}\right\}, \quad 0 \leqslant r_{1} \leqslant a_{1}
$$

$$
\mathbf{t}_{2}=\left\{\begin{array}{l}
\bar{\tau}_{r_{2 z_{2}}}\left(r_{2}, \theta_{2}\right) \\
\bar{\sigma}_{z_{2} z_{2}}\left(r_{2}, \theta_{2}\right) \\
\bar{\tau}_{\boldsymbol{\theta}_{2 z_{2}}}\left(r_{2}, \theta_{2}\right)
\end{array}\right\}=\sum_{n=0}^{\infty} \overline{\mathbf{L}}_{n}^{\mathrm{s}}\left\{\begin{array}{l}
\bar{\tau}_{\boldsymbol{\tau}_{2} z_{2}, n}^{\mathrm{s}}\left(r_{2}\right) \\
\bar{\sigma}_{z_{2} z_{2}, n}^{\mathrm{s}}\left(r_{2}\right) \\
\bar{\tau}_{\theta_{2} z_{2}, n}^{\mathrm{s}}\left(r_{2}\right)
\end{array}\right\}=\overline{\mathbf{L}}_{n}^{\mathrm{a}}\left\{\begin{array}{l}
\bar{\tau}_{\bar{\tau}_{2 z_{2}, n}}^{\mathrm{a}}\left(r_{2}\right) \\
\bar{\sigma}_{z_{2} z_{2}, n}^{\mathrm{a}}\left(r_{2}\right) \\
\bar{\tau}_{\theta_{2} z_{2}, n}^{\mathrm{a}}\left(r_{2}\right)
\end{array}\right\}, \quad 0 \leqslant r_{2} \leqslant a_{2}
$$

where diagonal matrices $\mathbf{L}_{n}^{\mathbf{s}}=\operatorname{diag}\left(\cos n \theta_{1}, \cos n \theta_{1},-\sin n \theta_{1}\right), \mathbf{L}_{n}^{\mathbf{a}}=\operatorname{diag}\left(\sin n \theta_{1}, \sin n \theta_{1}, \cos n \theta_{1}\right)$, super- 


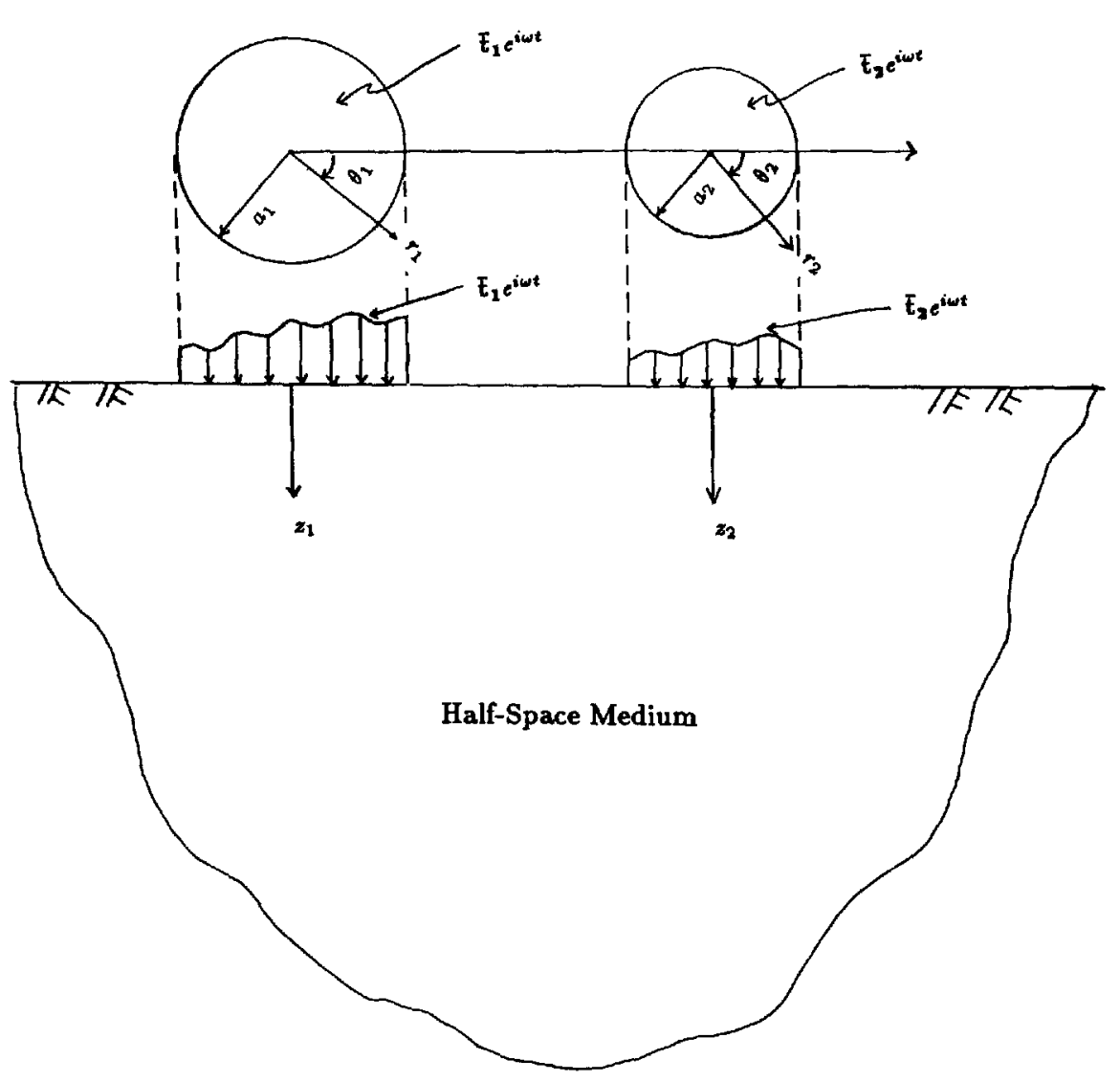

Figure 1. A half-space medium with two prescribed tractions

scripts $s$ and a denote symmetric and anti-symmetric with respect to the line of $\theta_{1}=0$ and $\theta_{2}=0$, and matrices $\overline{\mathbf{L}}_{n}^{\mathrm{s}}$ and $\overline{\mathbf{L}}_{n}^{\mathrm{a}}$ are similar to matrices $\mathbf{L}_{n}^{\mathrm{s}}$ and $\mathbf{L}_{n}^{\mathrm{a}}$ except that $\theta_{1}$ is replaced with $\theta_{2}$. In equations (1) and (2), the time harmonic variation $\mathrm{e}^{\mathrm{i} \omega t}$ has been omitted for convenience in later derivations.

For each Fourier component in equations (1) and (2), the tractions are assumed to be piecewice linear in the $r_{1}$ - and $r_{2}$-directions, respectively. Let the radius $a_{1}$ (for contact area No. 1) be divided into $m_{1}$ sub-intervals. One can express the traction of each Fourier component in the following form:

$$
\begin{aligned}
& \bar{\tau}_{r_{1} z_{1}, n}=\sum_{j=1}^{m_{1}-1} h_{j}\left(r_{1}\right) q_{j}+h_{o}\left(r_{1}\right) q_{o}+h_{m_{1}}\left(r_{1}\right) q_{m_{1}}=\mathbf{h}^{\mathrm{T}} \mathbf{q} \\
& \bar{\sigma}_{z_{1} z_{1}, n}=\sum_{j=1}^{m_{1}-1} h_{j}\left(r_{1}\right) p_{j}+h_{o}\left(r_{1}\right) p_{o}+h_{m_{1}}\left(r_{1}\right) p_{m_{1}}=\mathbf{h}^{\mathrm{T}} \mathbf{p} \\
& \bar{\tau}_{\theta_{1} z_{1}, n}=\sum_{j=1}^{m_{1}-1} h_{j}\left(r_{1}\right) s_{j}+h_{o}\left(r_{1}\right) s_{o}+h_{m_{1}}\left(r_{1}\right) s_{m_{1}}=\mathbf{h}^{\mathrm{T}} \mathbf{s}
\end{aligned}
$$

or

$$
\mathbf{t}_{1, n}\left(r_{1}\right)=\mathbf{H}_{1}^{\mathrm{T}} \mathbf{P}_{1, n}
$$


where

$h_{j}\left(r_{1}\right)= \begin{cases}1+\left(r_{1}-r_{1, j}\right) /\left(r_{1, j}-r_{1, j-1}\right) & \text { if } r_{1, j-1} \leqslant r_{1} \leqslant r_{1, j} \text { and } 1 \leqslant j \leqslant m_{1} \\ 1-\left(r_{1}-r_{1, j}\right) /\left(r_{1, j+1}-r_{1, j}\right) & \text { if } r_{1, j} \leqslant r_{1} \leqslant r_{1, j+1} \text { and } 0 \leqslant j \leqslant m_{1}-1 \\ 0 & \text { otherwise }\end{cases}$

matrix $\mathbf{H}_{1}^{\mathrm{T}}=\operatorname{diag}\left[\mathbf{h}^{\mathrm{T}}, \mathbf{h}^{\mathrm{T}}, \mathbf{h}^{\mathrm{T}}\right]$, vector $\mathbf{P}_{1, n}^{\mathrm{T}}=\left(\mathbf{q}^{\mathrm{T}}, \mathbf{p}^{\mathrm{T}}, \mathbf{s}^{\mathrm{T}}\right)$, and $q_{j}, p_{j}$ and $s_{j}$ are the stress intensities at nodal ring $j$ for $\bar{\tau}_{r_{1} z_{1}, n}, \bar{\sigma}_{z_{1} z_{1}, n}$ and $\bar{\tau}_{\theta_{1} z_{1}, n}$, respectively. Vector $\mathbf{P}_{1, n}$ in equation (3) could be either $\mathbf{P}_{1, n}^{\mathbf{s}}$ or $\mathbf{P}_{1, n}^{\text {a }}$ for symmetric or anti-symmetric Fourier components, respectively.

Similarly, the radius $a_{2}$ (of contact area No. 2) can be divided into $m_{2}$ sub-intervals and the traction of every Fourier component in equation (2) can be written as

$$
\mathbf{t}_{2, n}=\mathbf{H}_{2}^{\mathrm{T}} \mathbf{P}_{2, n}
$$

It should be noted that $\mathbf{H}_{1}^{\mathrm{T}}$ and $\mathbf{H}_{2}^{\mathrm{T}}$ are $3 \times 3\left(m_{1}+1\right)$ and $3 \times 3\left(m_{2}+1\right)$ matrices, respectively.

Consider the prescribed tractions of equation (1) with (3) only. References 1-3 reported an analytical solution for 3-D equations of wave propagation in a half-space medium satisfying this boundary condition. Using the analytical solution, the displacements at the surface of a half-space medium can be expressed as follows:

$$
\mathbf{u}_{1}\left(\mathbf{r}_{1}, \theta_{1}\right)=\sum_{n=0}^{\infty} \mathbf{L}_{n}^{\mathrm{s}} \int_{0}^{x} \mathbf{J}_{n} \mathbf{Q} \overline{\mathbf{D}}_{n} \mathbf{P}_{1, n}^{\mathrm{s}} \mathrm{d} k_{1}+\mathbf{L}_{n}^{\mathrm{a}} \int_{0}^{x} \mathbf{J}_{n} \mathbf{Q} \overline{\mathbf{D}}_{n} \mathbf{P}_{1, n}^{\mathrm{a}} \mathrm{d} k_{1}
$$

where

$$
\begin{aligned}
& \mathbf{J}_{n}=\left[\begin{array}{ccc}
J_{n}^{\prime}\left(k_{1} r_{1}\right) & 0 & \left(n / r_{1}\right) J_{n}\left(k_{1} r_{1}\right) \\
0 & k_{1} J_{n}\left(k_{1} r_{1}\right) & 0 \\
\left(n / r_{1}\right) J_{n}\left(k_{1} r_{1}\right) & 0 & J_{n}^{\prime}\left(k_{1} r_{1}\right)
\end{array}\right] \\
& \mathbf{Q}=\left[\begin{array}{ccc}
-v^{\prime} k_{\beta}^{2} / \Lambda & k_{1}\left(2 v v^{\prime}-2 k_{1}^{2}+k_{\beta}^{2}\right) / \Lambda & 0 \\
k_{1}\left(2 v v^{\prime}-2 k_{1}^{2}+k_{\beta}^{2}\right) / \Lambda & -v k_{\beta}^{2} / \Lambda & 0 \\
0 & 0 & -1 / G v^{\prime}
\end{array}\right] \\
& \Lambda=G\left[4 k_{1}^{2} v v^{\prime}-\left(2 k_{1}^{2}-k_{\beta}^{2}\right)^{2}\right] \\
& \overline{\mathbf{D}}_{n}=\left[\begin{array}{ccc}
\left(-\mathbf{D}_{n+1}^{\mathrm{T}}+\mathbf{D}_{n-1}^{\mathrm{T}}\right) / 2 & 0 & \left(\mathbf{D}_{n+1}^{\mathrm{T}}+\mathbf{D}_{n-1}^{\mathrm{T}}\right) / 2 \\
0 & \mathbf{D}_{n}^{\mathrm{T}} & 0 \\
\left(\mathbf{D}_{n+1}^{\mathrm{T}}+\mathbf{D}_{n-1}^{\mathrm{T}}\right) / 2 & 0 & \left(-\mathbf{D}_{n+1}^{\mathrm{T}}+\mathbf{D}_{n-1}^{\mathrm{T}}\right) / 2
\end{array}\right] \\
& \mathbf{D}_{n+1}^{\mathrm{T}}=\int_{0}^{a_{1}} r_{1} J_{n+1}\left(k_{1} r_{1}\right) \mathbf{h}^{\mathrm{T}} \mathrm{d} r_{\mathrm{l}} \\
& \mathbf{D}_{n}^{\mathrm{T}}=\int_{0}^{a_{1}} r_{1} J_{n}\left(k_{1} r_{1}\right) \mathbf{h}^{\mathrm{T}} \mathrm{d} r_{1} \\
& \mathbf{D}_{n-1}^{\mathrm{T}}=\int_{0}^{a_{1}} r_{1} J_{n-1}\left(k_{1} r_{1}\right) \mathbf{h}^{\mathrm{T}} \mathrm{d} r_{1}
\end{aligned}
$$

$G$ is the complex shear modulus of the half-space material,

$$
v=\sqrt{k_{1}^{2}-\omega^{2} / c_{\mathrm{p}}^{2}}, \quad v^{\prime}=\sqrt{k_{1}^{2}-\omega^{2} / c_{\mathrm{s}}^{2}}
$$

$c_{\mathrm{p}}$ and $c_{\mathrm{s}}$ are the compressional and the shear wave velocities in the half-space medium, $k_{1}$ the azimuthal wave number in $r_{1}, \theta_{1}, z_{1}$ co-ordinates, $J_{n}\left(k_{1} r_{1}\right)$ is the first kind of Bessel function of order $n$ and 
$J_{n}^{\prime}\left(k_{1} r_{1}\right)=\mathrm{d} J_{n}\left(k_{1} r_{1}\right) / \mathrm{d} r_{1}$. Note also that vectors $\mathbf{D}_{n+1}^{\mathrm{T}}, \mathbf{D}_{n}^{\mathrm{T}}$ and $\mathbf{D}_{n-1}^{\mathrm{T}}$ are Hankel transforms of the vector $\mathbf{h}^{\mathrm{T}}$ in equation (3).

Similarly, the analytical solution for 3-D equations of wave propagation satisfying the boundary condition of equation (2) with equation (4) can be obtained, except that the co-ordinate system is $r_{2}, \theta_{2}, z_{2}$ in Figure 1. The displacement vector at the surface of the half-space medium can be expressed as follows:

$$
\mathbf{u}_{2}=\sum_{m=0}^{\infty} \overline{\mathbf{L}}_{m}^{s} \int_{0}^{\infty} \overline{\mathbf{J}}_{m} \overline{\mathbf{Q}}_{\overline{\mathbf{D}}} \mathbf{P}_{2, m}^{\mathbf{s}} \mathrm{d} k_{2}+\overline{\mathbf{L}}_{m}^{\mathbf{a}} \int_{0}^{\infty} \overline{\mathbf{J}}_{m} \overline{\mathbf{Q}} \overline{\mathbf{D}}_{m} \mathbf{P}_{2, m}^{\mathbf{a}} \mathrm{d} k_{2}
$$

where matrices $\overline{\mathbf{J}}_{m}, \overline{\mathbf{Q}}$ and $\overline{\mathbf{D}}_{m}$ are similar to those defined in equation (5) except that $\boldsymbol{r}_{1}$ and $k_{1}$ are replaced with $r_{2}$ and $k_{2}$, respectively.

The principle of superposition is employed to obtain the total solution for the prescribed tractions of both equations (1) and (2). This leads to the total displacement vector at the surface of the half-space medium as follows:

$$
\mathbf{u}=\mathbf{u}_{1}+\mathbf{u}_{2}
$$

In equation (7), $\mathbf{u}_{1}$ is described by $r_{1}, \theta_{1}$ and $\mathbf{u}_{2}$ by $r_{2}, \theta_{2}$. Therefore, the co-ordinate transformation matrix must be introduced when the variational principle is employed to generate a dynamic stiffness matrix.

Consider the half-space medium with the prescribed tractions of equations (1) and (2). The work done by variations of $\mathbf{t}_{1}$ and $\mathbf{t}_{2}$ is

$$
\delta W=\int_{0}^{a_{1}} \int_{0}^{2 \pi} \delta \mathbf{t}_{1}^{\mathrm{T}}\left(\mathbf{u}_{1}+\mathbf{T} \mathbf{u}_{2}\right) r_{1} \mathrm{~d} \theta_{1} \mathrm{~d} r_{1}+\int_{0}^{a_{1}} \int_{0}^{2 \pi} \delta \mathbf{t}_{2}^{\mathbf{T}}\left(\mathbf{T}^{\mathrm{T}} \mathbf{u}_{1}+\mathbf{u}_{2}\right) r_{2} \mathrm{~d} \theta_{2} \mathrm{~d} r_{2}
$$

where $\mathbf{T}$ (referring to Figure 2) is the co-ordinate transformation matrix and can be expressed as follows:

$$
\mathbf{T}=\left[\begin{array}{ccc}
\cos \left(\theta_{2}-\theta_{1}\right) & 0 & -\sin \left(\theta_{2}-\theta_{1}\right) \\
0 & 1 & 0 \\
\sin \left(\theta_{2}-\theta_{1}\right) & 0 & \cos \left(\theta_{2}-\theta_{1}\right)
\end{array}\right]
$$

Replacing equation (1) with (3), equation (2) with (4) and substituting equations (5) and (6) into equation (8), and making use of the orthogonal property of Fourier components, equation (8) can be written, after some mathematical manipulations, as

$$
\begin{aligned}
\delta W= & \sum_{n=0}^{\infty}\left(\delta\left(\mathbf{P}_{1, n}^{\mathrm{s}}\right)^{\mathrm{T}} \mathbf{K}_{m n}^{\mathrm{s}} \mathbf{P}_{1, n}^{\mathrm{s}}+\delta\left(\mathbf{P}_{1, n}^{\mathrm{a}}\right)^{\mathrm{T}} \mathbf{K}_{m n}^{\mathrm{a}} \mathbf{P}_{1, n}^{\mathrm{a}}\right) \\
& +\sum_{n=0}^{\infty}\left(\delta\left(\mathbf{P}_{1, n}^{\mathrm{s}}\right)^{\mathrm{T}} \sum_{m=0}^{\infty} \mathbf{K}_{m, m}^{\mathrm{s}} \mathbf{P}_{2, m}^{\mathrm{s}}\right)+\left(\delta\left(\mathbf{P}_{1, n}^{\mathrm{a}}\right)^{\mathrm{T}} \sum_{m=0}^{\infty} \mathbf{K}_{n, m}^{\mathrm{a}} \mathbf{P}_{2, m}^{\mathrm{a}}\right) \\
& +\sum_{m=0}^{\infty}\left(\delta\left(\mathbf{P}_{2, m}^{\mathrm{s}}\right)^{\mathrm{T}} \overline{\mathbf{K}}_{m m}^{\mathrm{s}} \mathbf{P}_{2, m}^{\mathrm{s}}+\delta\left(\mathbf{P}_{2, m}^{\mathrm{a}}\right)^{\mathrm{T}} \overline{\mathbf{K}}_{m m}^{\mathrm{a}} \mathbf{P}_{2, m}^{\mathrm{a}}\right) \\
& +\sum_{m=0}^{\infty}\left(\delta\left(\mathbf{P}_{2, m}^{\mathrm{s}}\right)^{\mathrm{T}} \sum_{n=0}^{\infty} \mathbf{K}_{m, n}^{\mathrm{s}} \mathbf{P}_{1, n}^{\mathrm{s}}\right)+\left(\delta\left(\mathbf{P}_{2, m}^{\mathrm{a}}\right)^{\mathrm{T}} \sum_{n=0}^{\infty} \mathbf{K}_{m, n}^{\mathrm{a}} \mathbf{P}_{1, n}^{\mathrm{a}}\right)
\end{aligned}
$$

where

$$
\begin{aligned}
\mathbf{K}_{n n}^{\mathrm{s}} & =\int_{0}^{\infty} \int_{0}^{a_{1}} \int_{0}^{2 \pi} \mathbf{H}_{1} \mathbf{L}_{n}^{\mathrm{s}} \mathbf{L}_{n}^{\mathrm{s}} \mathbf{J}_{n} r_{1} \mathrm{~d} \theta_{1} \mathrm{~d} r_{1} \mathbf{Q} \overline{\mathbf{D}}_{n} \mathrm{~d} k_{1} \\
\mathbf{K}_{n n}^{\mathrm{a}} & =\int_{0}^{\infty} \int_{0}^{a_{1}} \int_{0}^{2 \pi} \mathbf{H}_{1} \mathbf{L}_{n}^{\mathrm{a}} \mathbf{L}_{n}^{\mathrm{a}} \mathbf{J}_{n} r_{1} \mathrm{~d} \theta_{1} \mathrm{~d} r_{1} \mathbf{Q} \overline{\mathbf{D}}_{n} \mathrm{~d} k_{1} \\
\mathbf{K}_{n, m}^{\mathrm{s}} & =\int_{0}^{\infty} \int_{0}^{a_{1}} \int_{0}^{2 \pi} \mathbf{H}_{1} \mathbf{L}_{n}^{\mathrm{s}} \mathbf{T} \overline{\mathbf{L}}_{m}^{\mathrm{s}} \overline{\mathbf{J}}_{m} r_{1} \mathrm{~d} \theta_{1} \mathrm{~d} r_{1} \overline{\mathbf{Q}} \overline{\mathbf{D}}_{m} \mathrm{~d} k_{2}
\end{aligned}
$$




$$
\begin{aligned}
\mathbf{K}_{n, m}^{\mathrm{a}} & =\int_{0}^{\infty} \int_{0}^{a_{1}} \int_{0}^{2 \pi} \mathbf{H}_{1} \mathbf{L}_{n}^{\mathrm{a}} \mathbf{T} \overline{\mathbf{L}}_{m}^{\mathrm{a}} \overline{\mathbf{J}}_{m} r_{1} \mathrm{~d} \theta_{1} \mathrm{~d} r_{1} \overline{\mathbf{Q}} \overline{\mathbf{D}}_{m} \mathrm{~d} k_{2} \\
\overline{\mathbf{K}}_{m m}^{\mathrm{s}} & =\int_{0}^{\infty} \int_{0}^{a_{2}} \int_{0}^{2 \pi} \mathbf{H}_{2} \overline{\mathbf{L}}_{m}^{\mathrm{s}} \overline{\mathbf{L}}_{m}^{\mathrm{s}} \overline{\mathbf{J}}_{m} r_{2} \mathrm{~d} \theta_{2} \mathrm{~d} r_{2} \overline{\mathbf{Q}} \overline{\mathbf{D}}_{m} \mathrm{~d} k_{2} \\
\overline{\mathbf{K}}_{m m}^{\mathrm{a}} & =\int_{0}^{\infty} \int_{0}^{a_{2}} \int_{0}^{2 \pi} \mathbf{H}_{2} \overline{\mathbf{L}}_{m}^{\mathrm{a}} \overline{\mathbf{L}}_{m}^{\mathrm{a}} \overline{\mathbf{J}}_{m} r_{2} \mathrm{~d} \theta_{2} \mathrm{~d} r_{2} \overline{\mathbf{Q}} \overline{\mathbf{D}}_{m} \mathrm{~d} k_{2} \\
\overline{\mathbf{K}}_{m, n}^{\mathrm{s}} & =\int_{0}^{\infty} \int_{0}^{a_{2}} \int_{0}^{2 \pi} \mathbf{H}_{2} \overline{\mathbf{L}}_{m}^{\mathrm{s}} \mathbf{T}^{\mathrm{T}} \mathbf{L}_{n}^{\mathrm{s}} \overline{\mathbf{J}}_{n} r_{2} \mathrm{~d} \theta_{2} \mathrm{~d} r_{2} \mathbf{Q} \mathbf{D}_{n} \mathrm{~d} k_{1} \\
\overline{\mathbf{K}}_{m, n}^{\mathrm{a}} & =\int_{0}^{\infty} \int_{0}^{a_{2}} \int_{0}^{2 \pi} \mathbf{H}_{2} \overline{\mathbf{L}}_{m}^{\mathrm{a}} \mathbf{T}^{\mathrm{T}} \mathbf{L}_{n}^{\mathrm{a}} \mathbf{J}_{n} r_{2} \mathrm{~d} \theta_{2} \mathrm{~d} r_{2} \mathbf{Q} \mathbf{D}_{m} \mathrm{~d} k_{1}
\end{aligned}
$$

In equation (9), the integral of the product of symmetric and anti-symmetric Fourier components with respect to the line $\theta_{1}=0$ and $\theta_{2}=0$ (Figure 1) has been set equal to zero.

If one truncates the infinite Fourier series of equations (1) and (2) to finite series, and let $A$ and $B$ be the highest components for equations (1) and (2), respectively, equation (9) can be written in a matrix form as

$$
\delta W=\left[\begin{array}{l}
\delta \mathbf{P}^{\mathrm{s}} \\
\delta \mathbf{P}^{\mathrm{a}}
\end{array}\right]^{\mathrm{T}}\left[\begin{array}{lr}
\mathbf{K}^{\mathrm{s}} & 0 \\
0 & \mathbf{K}^{\mathrm{a}}
\end{array}\right]\left[\begin{array}{l}
\mathbf{P}^{\mathrm{s}} \\
\mathbf{P}^{\mathrm{a}}
\end{array}\right]
$$

where

$$
\begin{gathered}
\mathbf{P}^{\mathrm{s}^{\mathrm{T}}}=\left[\begin{array}{llllll}
\mathbf{P}_{1,1}^{\mathrm{s}} & \cdots & \mathbf{P}_{1, A}^{\mathrm{s}^{\mathrm{T}}} & \mathbf{P}_{2,1}^{\mathrm{s}^{\mathrm{T}}} & \cdots & \mathbf{P}_{2, B}^{\mathrm{T}}
\end{array}\right] \\
\mathbf{P}^{\mathrm{a}^{\mathrm{T}}}=\left[\begin{array}{lllllll}
\mathbf{P}_{1,1}^{\mathrm{a}^{\mathrm{T}}} & \cdots & \mathbf{P}_{1, A}^{\mathrm{a}^{\mathrm{T}}} & \mathbf{P}_{2,1}^{\mathrm{a}^{\mathrm{T}}} & \cdots & \mathbf{P}_{2, B}^{\mathrm{a}^{\mathrm{T}}}
\end{array}\right] \\
\mathbf{K}^{\mathrm{s}}=\left[\begin{array}{ccccccc}
\mathbf{K}_{11}^{\mathrm{s}} & 0 & \cdots & 0 & \mathbf{K}_{1,1}^{\mathrm{s}} & \cdots & \mathbf{K}_{1, B}^{\mathrm{s}} \\
0 & \mathbf{K}_{22}^{\mathrm{s}} & \cdots & 0 & \mathbf{K}_{2.1}^{\mathrm{s}} & \cdots & \mathbf{K}_{2, B}^{\mathrm{s}} \\
\vdots & \vdots & \ddots & \vdots & \vdots & \ddots & \vdots \\
0 & 0 & \cdots & \mathbf{K}_{A,}^{\mathrm{s}} & \mathbf{K}_{A, 1}^{\mathrm{s}} & \cdots & \mathbf{K}_{A, B}^{\mathrm{s}} \\
\overline{\mathbf{K}}_{1.1}^{\mathrm{s}} & \overline{\mathbf{K}}_{1,2}^{\mathrm{s}} & \cdots & \overline{\mathbf{K}}_{1, A}^{\mathrm{s},} & \overline{\mathbf{K}}_{1,1}^{\mathrm{s}} & \cdots & 0 \\
\vdots & \vdots & \vdots & \vdots & \vdots & \ddots & \vdots \\
\overline{\mathbf{K}}_{B, 1}^{\mathrm{s}} & \overline{\mathbf{K}}_{B, 2}^{\mathrm{s}} & \cdots & \overline{\mathbf{K}}_{B, A}^{\mathrm{s}} & 0 & \cdots & \overline{\mathbf{K}}_{B, B}^{\mathrm{s}}
\end{array}\right]
\end{gathered}
$$

and the expression for $\mathbf{K}^{\mathrm{a}}$ is similar to that for $\mathbf{K}^{\mathrm{s}}$. From equation (14), one can observe that the interactions among Fourier components only occur between two symmetric components and between two antisymmetric components, and there are no interactions between symmetric and anti-symmetric components with respect to the line $\theta_{1}=0$ and $\theta_{2}=0$ in Figure 1. Also, by reciprocal theorem (Betti's theorem), one can easily prove that $\overline{\mathbf{K}}_{n n}^{\mathrm{s}}=\left(\mathbf{K}_{n n}^{\mathrm{s}}\right)^{\mathrm{T}}, \overline{\mathbf{K}}_{n n}^{\mathrm{a}}=\left(\mathbf{K}_{n n}^{\mathrm{a}}\right)^{\mathrm{T}}, \mathbf{K}_{n, m}^{\mathrm{s}}=\left(\overline{\mathbf{K}}_{m, n}^{\mathrm{s}}\right)^{\mathrm{T}}$ and $\mathbf{K}_{n, m}^{\mathrm{a}}=\left(\overline{\mathbf{K}}_{m, n}^{\mathrm{a}}\right)^{\mathrm{T}}$. Therefore both $\mathbf{K}^{\mathrm{s}}$ and $\mathbf{K}^{\mathrm{a}}$ are symmetric matrices.

Now consider the sub-structure of the two foundations. If finite element modelling is employed for the sub-structure, the displacement fields of the two foundations can be expressed as

and

$$
\mathbf{v}_{1}\left(r_{1}, \theta_{1}\right)=\sum_{n=0}^{A}\left[\mathbf{L}_{n}^{\mathrm{s}} \mathbf{N}_{1}^{\mathrm{s}}\left(r_{1}\right) \mathbf{V}_{1 . n}^{\mathrm{s}}+\mathbf{L}_{n}^{\mathrm{a}} \mathbf{N}_{1}^{\mathrm{a}}\left(r_{1}\right) \mathbf{V}_{1, n}^{\mathrm{a}}\right]
$$

$$
\mathbf{v}_{2}\left(r_{2}, \theta_{2}\right)=\sum_{m=0}^{B}\left[\overline{\mathbf{L}}_{m}^{\mathrm{s}} \mathbf{N}_{2}^{\mathrm{s}}\left(r_{2}\right) \mathbf{V}_{2, m}^{\mathrm{s}}+\overline{\mathbf{L}}_{m}^{\mathrm{a}} \mathbf{N}_{2}^{\mathrm{a}}\left(r_{2}\right) \mathbf{V}_{2, m}^{\mathrm{a}}\right]
$$

where $\mathbf{N}_{1}$ and $\mathbf{N}_{2}$ are the matrices of the shape functions with respect to the variables $r_{1}$ and $r_{2}$, respectively. 
vectors $\mathbf{V}_{1, n}$ and $\mathbf{V}_{2, m}$ are the displacements at the nodal rings of the finite element model, and matrices $\mathbf{L}_{n}$ and $\overline{\mathbf{L}}_{m}$ are defined in equations (1) and (2). Making use of equations (15) and (16), the work done by the variation of $t_{1}$ and $t_{2}$ in equations (1) and (2) can be expressed the following equation.

$$
\begin{aligned}
\delta W & =\int_{0}^{a_{1}} \int_{0}^{2 \pi} \delta \mathbf{t}_{1}^{\mathrm{T}} \mathbf{v}_{1} r_{1} \mathrm{~d} \theta_{1} \mathrm{~d} r_{1}+\int_{0}^{a_{2}} \int_{0}^{2 \pi} \delta \mathbf{t}_{2}^{\mathrm{T}} \mathbf{v}_{2} r_{1} \mathrm{~d} \theta_{2} \mathrm{~d} r_{2} \\
& =\sum_{n=0}^{A}\left(\delta \mathbf{P}_{1, n}^{\mathbf{s}^{\mathrm{T}}} \mathbf{B}_{1, n}^{\mathrm{s}} \mathbf{V}_{1, n}^{\mathrm{s}}+\delta \mathbf{P}_{1, n}^{\mathrm{a}^{\mathrm{T}}} \mathbf{B}_{1, n}^{\mathrm{a}} \mathbf{V}_{1, n}^{\mathrm{a}}\right)+\sum_{m=0}^{B}\left(\delta \mathbf{P}_{2, m}^{\mathrm{s}^{\mathrm{T}}} \mathbf{B}_{2, m}^{\mathrm{s}} \mathbf{V}_{2, m}^{\mathrm{s}}+\delta \mathbf{P}_{2, m}^{\mathrm{a}^{\mathrm{T}}} \mathbf{B}_{2, m}^{\mathrm{a}} \mathbf{V}_{2, m}^{\mathrm{a}}\right)
\end{aligned}
$$

where

$$
\mathbf{B}_{1, n}=\int_{0}^{a_{1}} \int_{0}^{2 \pi} \mathbf{H}_{1} \mathbf{L}_{n} \mathbf{L}_{n} \mathbf{N}_{1} r_{1} \mathrm{~d} \theta_{1} \mathrm{~d} r_{1}
$$

the expression for $\mathbf{B}_{2, m}$ is similar to that in equation (17b), and matrices $\mathbf{H}_{1}$ and $\mathbf{L}_{n}$ are defined in equations (3) and (1). In a similar way to equation (14), the integration of the products of the symmetric and anti-symmetric Fourier components has also been set to be zero in equation (17), and the infinite Fourier series in equations (15) and (16) have also been truncated to finite series in order to make equation (17) compatible with equation (14). Equation (17) can also be rewritten in matrix form as

$$
\delta W=\left[\begin{array}{l}
\delta \mathbf{P}^{\mathrm{s}} \\
\delta \mathbf{P}^{\mathrm{a}}
\end{array}\right]^{\mathrm{T}}\left[\begin{array}{ll}
\mathbf{B}^{\mathrm{s}} & 0 \\
0 & \mathbf{B}^{\mathrm{a}}
\end{array}\right]\left[\begin{array}{c}
\mathbf{V}^{\mathrm{s}} \\
\mathbf{V}^{\mathrm{a}}
\end{array}\right]
$$

where

$$
\begin{aligned}
\delta \mathbf{P}^{\mathbf{s}^{\mathrm{T}}} & =\left[\begin{array}{llllll}
\delta \mathbf{P}_{1,1}^{\mathbf{s}^{\mathrm{T}}} & \cdots & \delta \mathbf{P}_{1, A}^{\mathbf{s}^{\mathrm{T}}} & \delta \mathbf{P}_{2,1}^{\mathbf{s}^{\mathrm{T}}} & \cdots & \delta \mathbf{P}_{2, \boldsymbol{B}}^{\mathbf{s}^{\mathrm{T}}}
\end{array}\right] \\
\mathbf{V}^{\mathbf{s}^{\mathbf{T}}} & =\left[\begin{array}{lllllll}
\mathbf{V}_{1,1}^{\mathbf{s}^{\mathrm{T}}} & \cdots & \mathbf{V}_{1, A}^{\mathbf{s}^{T}} & \mathbf{V}_{2,1}^{\mathbf{s}^{\mathrm{T}}} & \cdots & \mathbf{V}_{2, \boldsymbol{B}}^{\mathbf{s}^{\mathrm{T}}}
\end{array}\right] \\
\mathbf{B}^{\mathbf{s}} & =\operatorname{diag}\left[\begin{array}{lllllll}
\mathbf{B}_{1,1}^{\mathbf{s}} & \cdots & \mathbf{B}_{1, A}^{\mathbf{s}} & \mathbf{B}_{2,1}^{\mathbf{s}} & \cdots & \mathbf{B}_{2, \boldsymbol{B}}^{\mathrm{s}}
\end{array}\right]
\end{aligned}
$$

and expressions for vectors $\mathbf{P}^{\mathrm{a}}, \mathbf{V}^{\mathrm{a}}$ and matrix $\mathbf{B}^{\mathrm{a}}$ are similar to those for $\mathbf{P}^{\mathrm{s}}, \mathbf{V}^{\mathrm{s}}$ and $\mathbf{B}^{\mathrm{s}}$, respectively.

Equating equations (14) and (18), one can obtain the following two equations:

$$
\mathbf{K}^{\mathbf{s}} \mathbf{P}^{\mathrm{s}}=\mathbf{B}^{\mathrm{s}} \mathbf{V}^{\mathrm{s}} \text { or } \mathbf{U}^{\mathrm{s}}=\mathbf{B}^{\mathbf{s}} \mathbf{V}^{\mathbf{s}}
$$

and

$$
\mathbf{K}^{\mathbf{a}} \mathbf{P}^{\mathbf{a}}=\mathbf{B}^{\mathbf{a}} \mathbf{V}^{\mathbf{a}} \text { or } \mathbf{U}^{\mathrm{a}}=\mathbf{B}^{\mathbf{a}} \mathbf{V}^{\mathbf{a}}
$$

where vectors $\mathbf{U}^{s}$ and $\mathbf{U}^{\mathrm{a}}$ are the generalized displacements at the nodal rings of the assumed piecewise linear stress models of equations (1) and (2). Equations (19) and (20) give the relationship between the generalized displacements of the assumed stress model and the finite element model of equations (15) and (16). The reciprocal theorem can be used to find the corresponding force-stress relationship. This gives

$$
\mathbf{F}^{\mathbf{s}}==\mathbf{B}^{\mathbf{s}^{\mathrm{T}}} \mathbf{P}^{\mathbf{s}} \text { and } \quad \mathbf{F}^{\mathbf{a}}=\mathbf{B}^{\mathbf{a}^{\mathrm{T}}} \mathbf{P}^{\mathrm{a}}
$$

where the vectors $\mathbf{F}^{\mathrm{s}}$ and $\mathbf{F}^{\mathrm{a}}$ are the generalized forces at the nodal rings of the finite element models of equations (15) and (16). The following two equations can be obtained by making use of equations (19) and (20) with equation (21).

$$
\mathbf{F}^{\mathrm{s}}=\mathbf{B}^{\mathrm{s}^{\mathrm{T}}}\left[\mathbf{K}^{\mathrm{s}}\right]^{-1} \mathbf{B}^{\mathrm{s}} \mathbf{V}^{\mathrm{s}}=\mathbf{I}^{\mathrm{s}} \mathbf{V}^{\mathrm{s}}
$$

and

$$
\mathbf{F}^{\mathbf{a}}=\mathbf{B}^{\mathbf{a}^{\mathrm{T}}}\left[\mathbf{K}^{\mathrm{a}}\right]^{-1} \mathbf{B}^{\mathrm{a}} \mathbf{V}^{\mathrm{a}}=\mathbf{I}^{\mathrm{a}} \mathbf{V}^{\mathrm{a}}
$$

where $I^{s}$ is the dynamic stiffness matrix for the excitations of the symmetric Fourier components with respect to the line $\theta_{1}=0$ and $\theta_{2}=0$, as shown in Figure 2, and $\mathbf{I}^{\mathrm{a}}$ is the dynamic stiffness matrix for the excitations of 


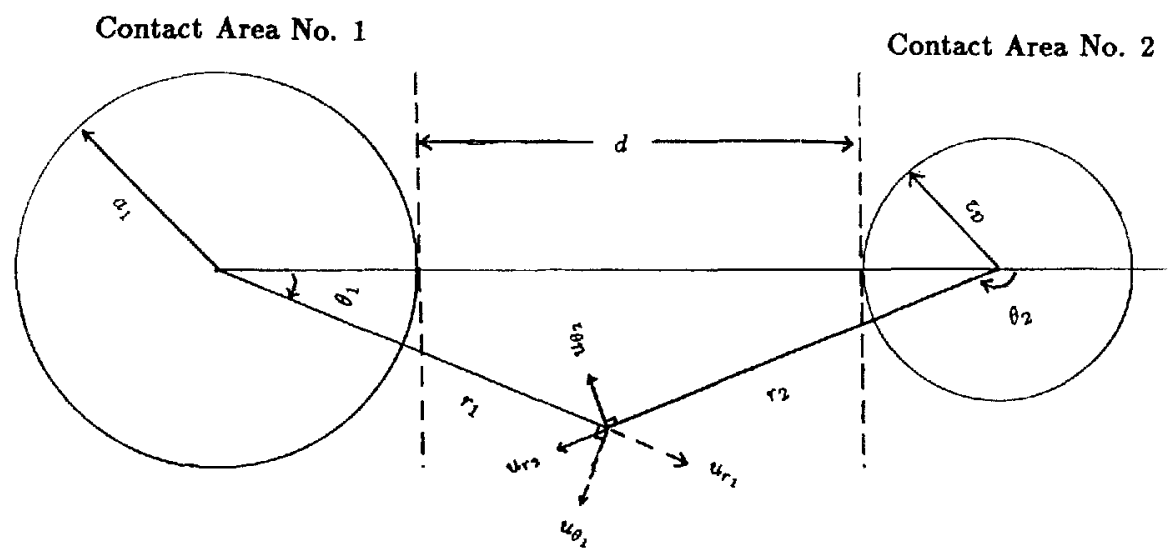

Figure 2. Co-ordinate transformation for two cylindrical co-ordinates

the anti-symmetric Fourier components. Both the $\mathbf{I}^{\mathbf{s}}$ and $\mathbf{I}^{\mathbf{a}}$ matrices are symmetric since matrices $\mathbf{K}^{\mathrm{s}}$ and $\mathbf{K}^{\mathrm{a}}$ are symmetric as indicated in equation (14). Equations (22) and (23) also show that there are no interactions between the symmetric vibration mode and the anti-symmetric vibration mode with respect to the line connecting the two centres of the two circular foundations (Figure 2).

This concludes the derivations for the dynamic stiffness matrices for the interaction of the vibrations of two circular foundations on an elastic half-space medium.

\section{NUMERICAL RESULTS}

A numerical example of two identical rigid circular foundations, rigidly attached to an elastic half-space medium and subjected to excitations in all possible directions, is used to demonstrate the effectiveness and efficiency of the present method. In the example, the radius of both foundations is $a_{0}\left(a_{0}=0.5\right)$, the complex shear modulus of the half-space is $G=G_{R}(1+2 \xi i)$, in which the hysteresis damping ratio $\xi$ is selected to be 0.05 for major numerical results, and the Poisson ratio is assumed to be 0.33 . In order to investigate the significance of the effect of the interaction of the two foundations, seven clear distances ( $d$ 's) $\left(d / a_{0}=0 \cdot 2,1,2,4,10,20\right.$ and $\left.\infty\right)$ between the two foundations are chosen for the calculation of dynamic stiffness matrices.

Since both circular foundations are rigid, only Fourier components of $n=0,1$ and $m=0,1$ in equations (22) and (23) are involved in the calculation of the dynamic stiffness matrix for all the vertical, two horizontal $(x$ - and $y$-directions in Figure 3), rocking, pitching and torsional vibrations of the two foundations, as defined in Figure 3. Equation (22) is the dynamic stiffness matrix for the two foundations with vertical, horizontal ( $x$-direction) and pitching motions. Equation (23) is the dynamic stiffness matrix for the two foundations with torsional, horizontal ( $y$-direction) and rocking motions. Furthermore, due to symmetry and anti-symmetry with respect to the centre line $C-C$ between the two foundations, as shown in Figure 3, the dynamic stiffness functions in the two dynamic stiffness matrices have the following properties: $I_{z_{1} z_{1}}=I_{z_{2} z_{2}}, I_{x_{1} x_{1}}=I_{x_{2} x_{2}}$. $I_{P_{1} P_{1}}=I_{P_{2} P_{2}}, I_{z_{1} x_{1}}=-I_{z_{2} x_{2}}, I_{z_{1} P_{1}}=-I_{z_{2} P_{2}}$ and $I_{x_{1} P_{1}}=I_{x_{2} P_{2}}$ for the dynamic stiffness matrix in equation (22), and $I_{T_{1} T_{1}}=I_{T_{2} T_{2}}, I_{y_{1} y_{1}}=I_{y_{2} y_{2}}, I_{R_{1} R_{1}}=I_{R_{2} R_{2}}, I_{T_{1} y_{1}}=-I_{T_{2} y_{2}}, I_{T_{1} R_{1}}=-I_{T_{2} R_{2}}$ and $I_{y_{1} R_{1}}=I_{y_{2} R_{2}}$ for the dynamic stiffness matrix in equation (23).

Using this method, one should note that singularity could occur in calculating the $\mathbf{Q}$ matrix of equation (5b) at the Rayleigh wave number if the damping ratio $\xi=0$. Therefore, some material damping, which would meet the realistic condition of the soil, must be assumed in the soil medium. After some extensive numerical investigations, the infinite integration limit of the wave number in equations 10 (a) 13(b) can be replaced with the finite number $200 / a_{0}$ without losing accuracy for a damping ratio $\xi$ as low as $0 \cdot 01$, and 10 equal sub-intervals for radius $a_{0}$ is enough for a piecewise linear stress model of equations (3) and (4). The Gaussian quadrature method is employed to perform the integrations for which closed-form integration cannot be obtained. 
In order to show the effectiveness of this method, some comparisons with the results in Reference 15 are made in Table I. As mentioned above, material damping in a soil medium is necessary, and the results for $\xi=0.01$ are used for comparison. In the table, the non-dimensionalized frequency $b$ is defined as $\omega a_{0} / \operatorname{Re}\left(c_{\mathrm{s}}\right)$. Although there are some discrepancies between the results of the two methods, in general one can still say that the two results agree fairly well with each other except $I_{P_{1} P_{1}}$, for which the discrepancy is higher. Since $I_{P_{1} P_{1}}, I_{x_{1} x_{1}}$ and $I_{z_{1} z_{1}}$ are obtained simultaneously by equation (22), as mentioned above, it is believed that the accuracy of these dynamic stiffnesses should be of the same order. The sources of the discrepancies could be the error due to digitizing the curves in Reference 15, and the use of two different damping ratios for the soil medium and two different analytical approaches.

Figures 4-10 show some numerical results of dynamic stiffness functions for the vibrations of the two foundations. In the figures, it should be noted that all the dynamic stiffness functions and excitation frequencies are non-dimensionalized by radius $a_{0}$ and complex shear modulus $G$ [or shear wave velocity $\left.\operatorname{Re}\left(c_{\mathrm{s}}\right)\right]$. Each figure contains several curves of results representing different clear distances between the two foundations. The curves for different clear distances are marked by different symbols. Table II shows the symbols used.

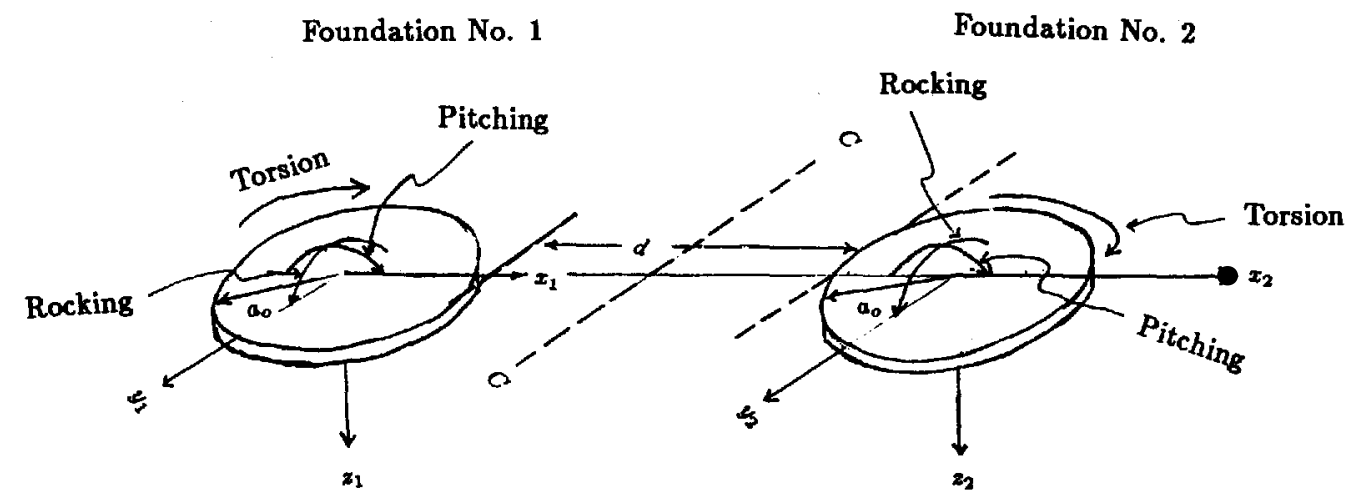

Figure 3. Definitions of the motions for two identical foundations

Table I. Comparison for clear distance $d=0.5 a_{0}$

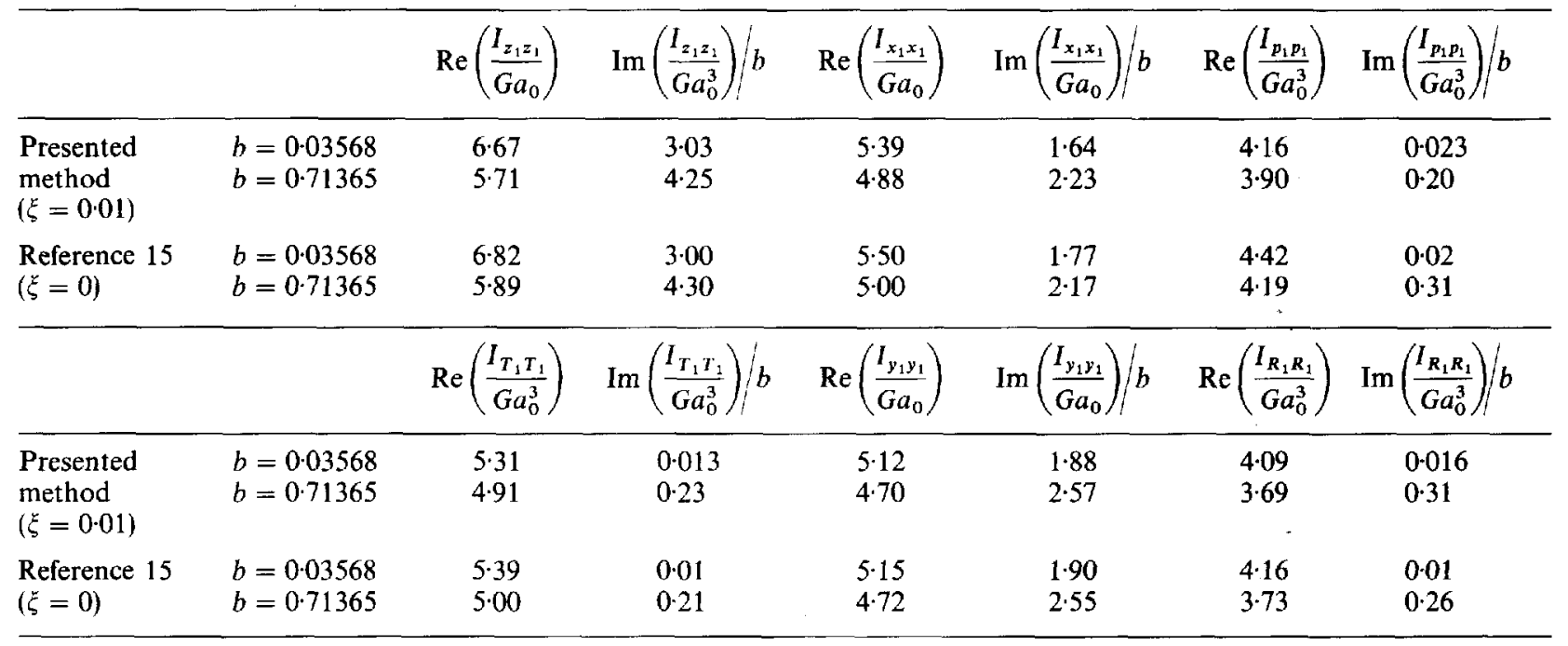


Table II. Symbols representing different separations of the two foundations

\begin{tabular}{|c|c|}
\hline $0-\theta-0$ & $\frac{d}{a_{o}}=0 \cdot 2$ \\
\hline$+1+1$ & $\frac{d}{a_{o}}=1$ \\
\hline$\leftrightarrow x \leftrightarrow x$ & $\frac{d}{a_{o}}=2$ \\
\hline$\leftrightarrow \theta \theta \theta$ & $\frac{d}{a_{0}}=4$ \\
\hline 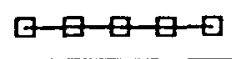 & $\frac{d}{a_{a}}=10$ \\
\hline$\Delta \Delta \Delta \Delta \Delta$ & $\frac{d}{a_{0}}=20$ \\
\hline$* * * * * *$ & $\frac{d}{a_{0}}=\infty$ \\
\hline
\end{tabular}

Figure 4 shows the dynamic stiffness function $\left(I_{z_{1} z_{1}}\right)$ for the vertical vibration of one of the two foundations. From the figure, it can be seen that the influence from the other foundation on the vertical dynamic stiffness is not significant if the separation of the two foundations is greater than $4 a_{0}\left(d / a_{0}>4\right.$ in Figure 4). Figure 5 gives the results of the horizontal dynamic stiffness function. In the figure, the solid lines show the results for the horizontal vibration in the $x$-direction and the broken lines show the results for vibration in the $y$-direction. The same conclusion can be drawn from this figure as from Figure 4, and it can also be seen that the influence of the existence of the other foundation is more important for the vibration in the $x$ - direction than for the vibration in the $y$-direction. Regarding the dynamic stiffness for pitching and rocking motions, the influence from other foundation is even less than that for the horizontal dynamic stiffness as shown in Figure 6. The solid lines in Figure 6 are the results of pitching dynamic stiffness and the broken lines are the results of rocking dynamic stiffness. From Figure 6 , it can also be seen that the influence on the pitching dynamic stiffness from the other foundation only occurs when the two foundations are sufficiently close (saying $d / a_{0} \leqslant 1 \cdot 0$ ), and the influence on the rocking dynamic stiffness is insignificant (the broken lines cannot be distinguished from the solid lines). Figure 7 shows the coupling dynamic stiffness for pitching and horizontal motions (solid lines), and rocking and horizontal motions (broken lines). From this figure, it can be seen that the influence from the other foundation may be significant if the two foundations are very close (say $d / a_{0} \leqslant 1 \cdot 0$ ). The reason for putting the two horizontal dynamic stiffnesses (Figure 5), the pitching and rocking dynamic stiffnesses (Figure 6) and the two coupling dynamic stiffnesses (Figure 7) together is that in each case they are identical to each other if the two foundations are infinitely far apart. Figure 8 shows the dynamic stiffness function for the torsional vibration. From this figure, it can be seen that the influence on the torsional dynamic stiffness from the other foundation is unimportant even when the two foundations are very close $\left(d / a_{0}=0 \cdot 2\right)$. Figure 9 shows the coupling dynamic stiffness function between the vertical vibrations of the two foundations. It can be seen that the coupling dynamic stiffness function approaches zero as $d / a_{0}=20$ or $\propto$. A similar result can be seen in Figure 10, which shows the coupling dynamic stiffness between the torsional vibrations of the two foundations. From Figures 9 and 10, it can be seen that the coupling dynamic stiffnesses between the vibrations of two foundations cannot be neglected in soil-structure interaction analysis, especially when the two foundations are close to each other.

After some numerical study of the dynamic stiffness matrices for the two-foundation system, some general conclusions can be drawn.

1. The interaction of the vibrations of two foundations with an excitation of low frequency is more important than that with an excitation of high frequency.

2. The influence on the imaginary parts of the dynamic stiffness functions due to the existence of another foundation is less significant. 


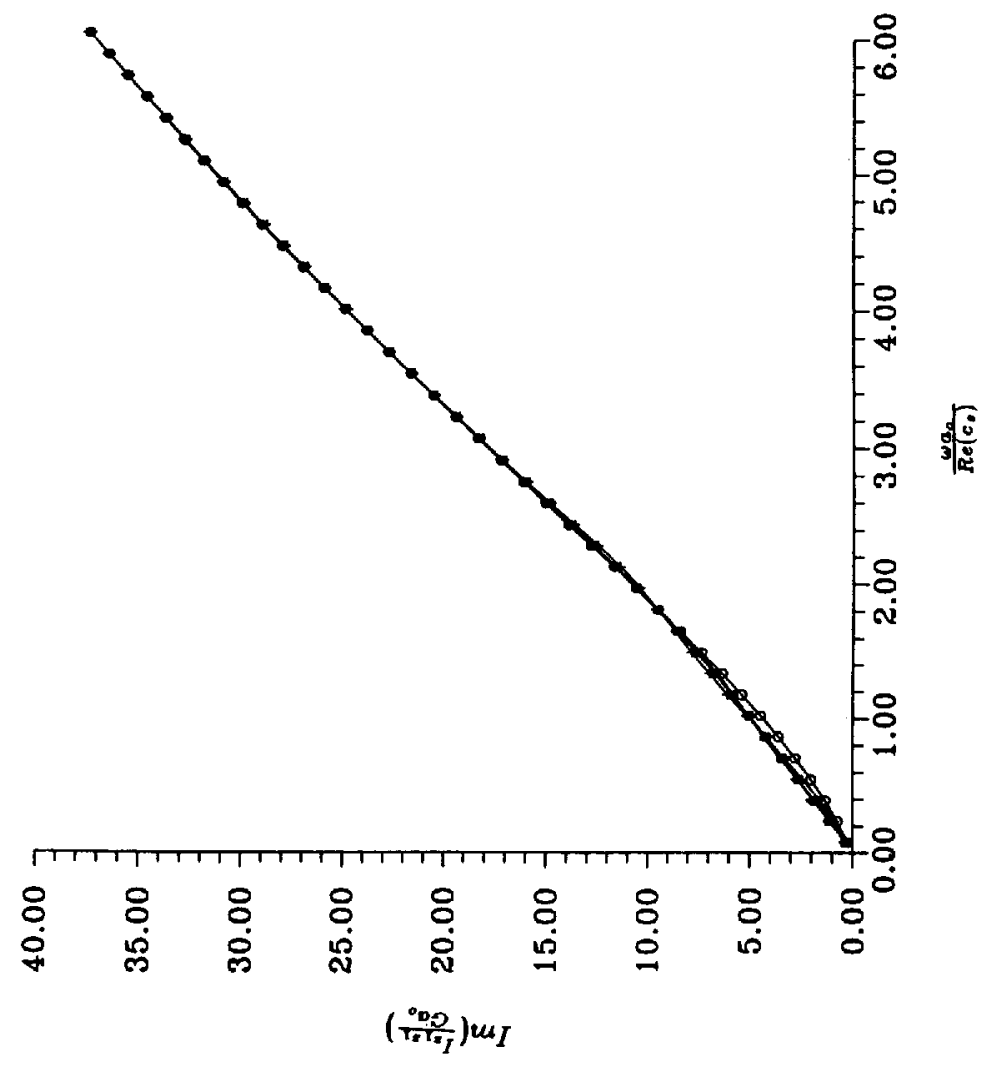

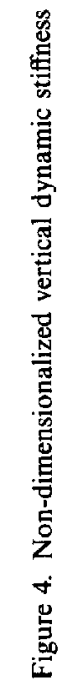

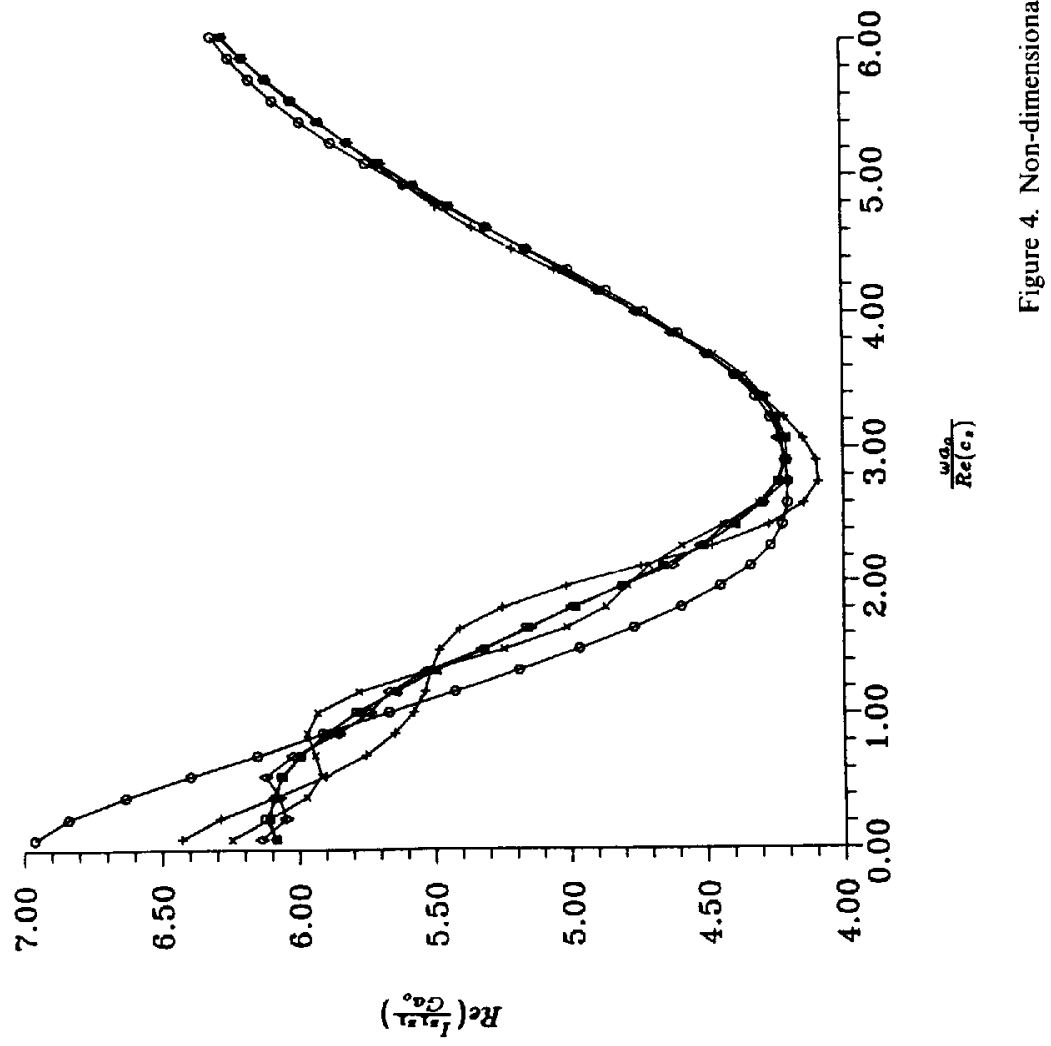




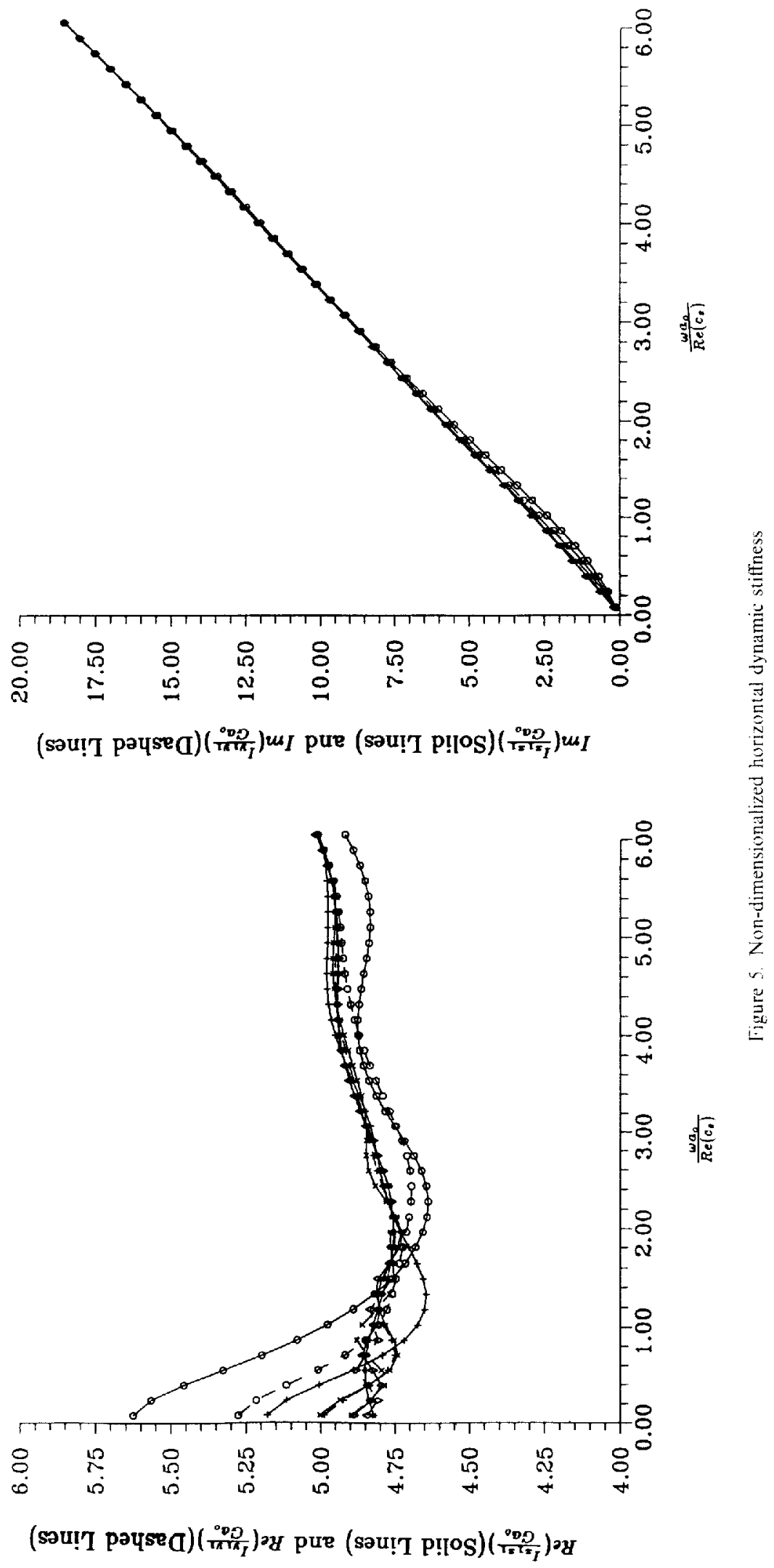




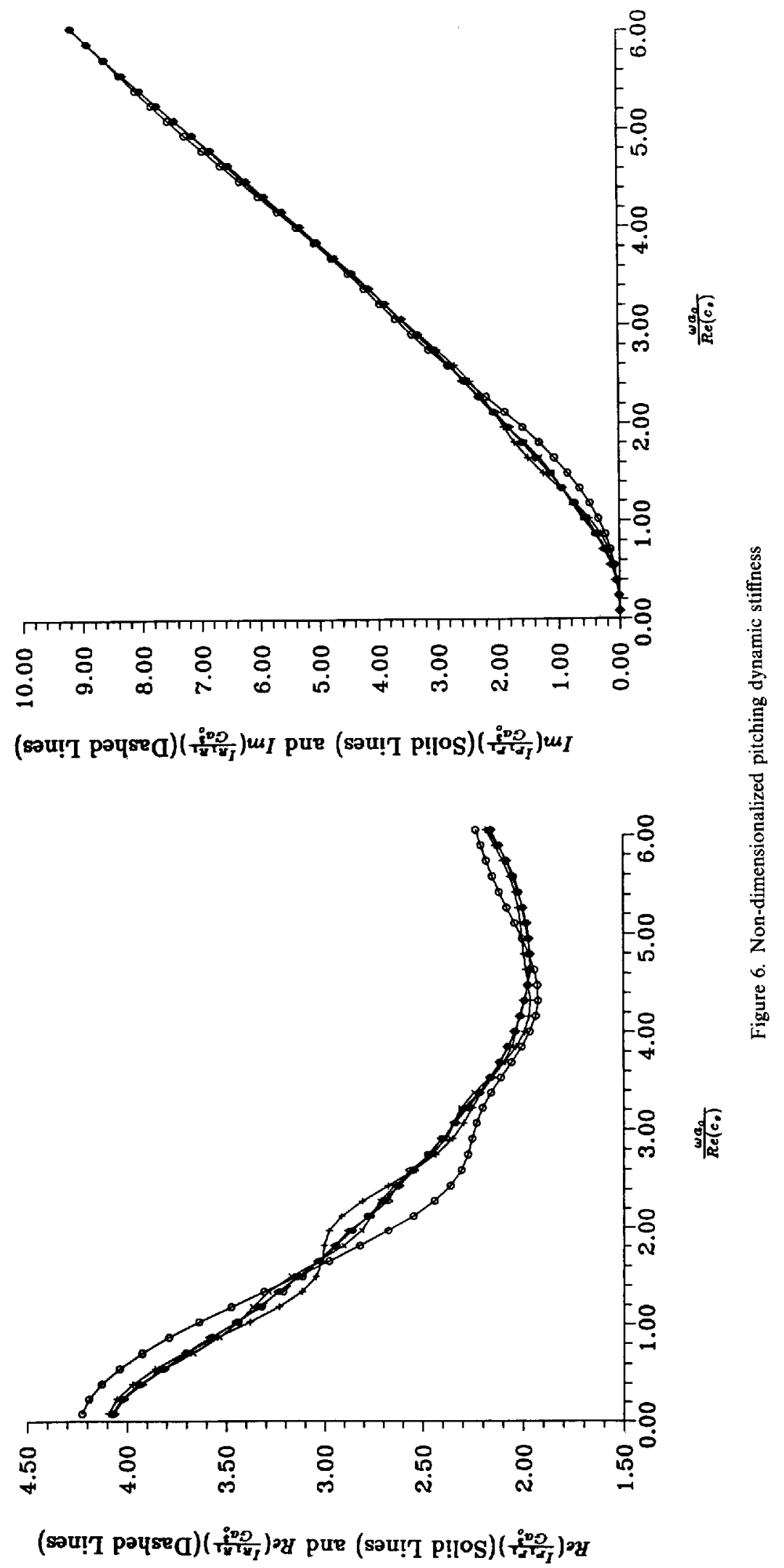



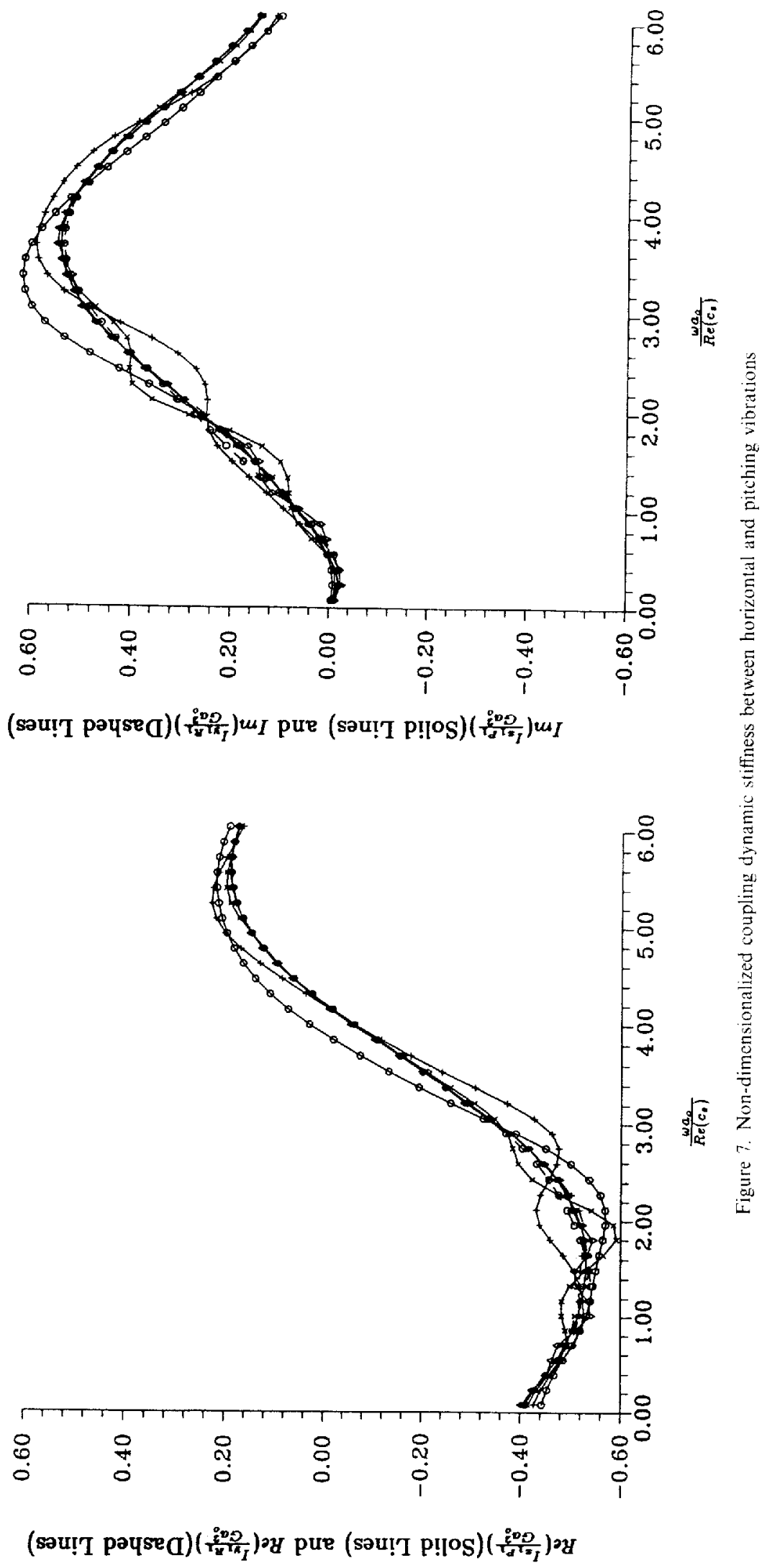


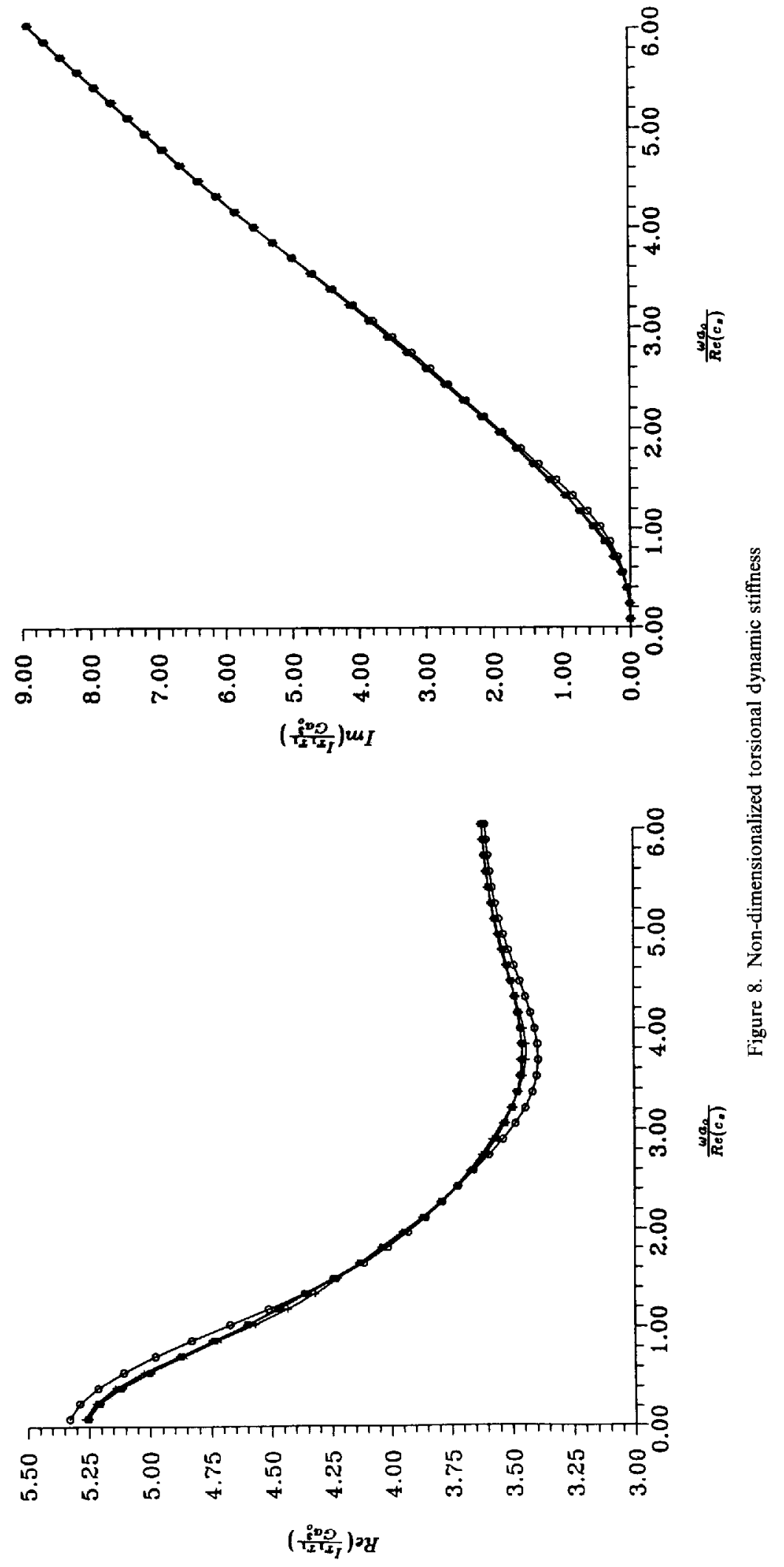



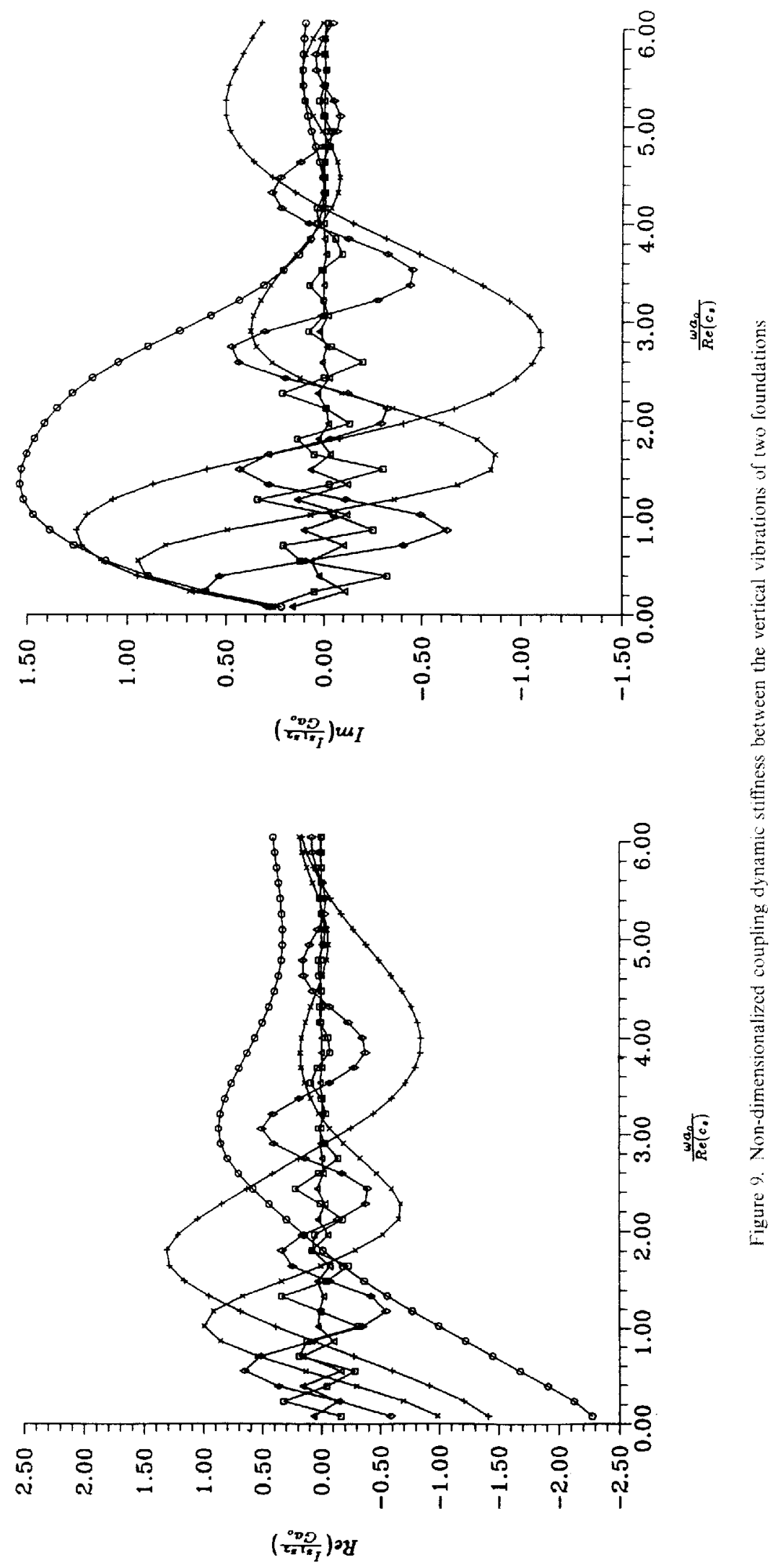

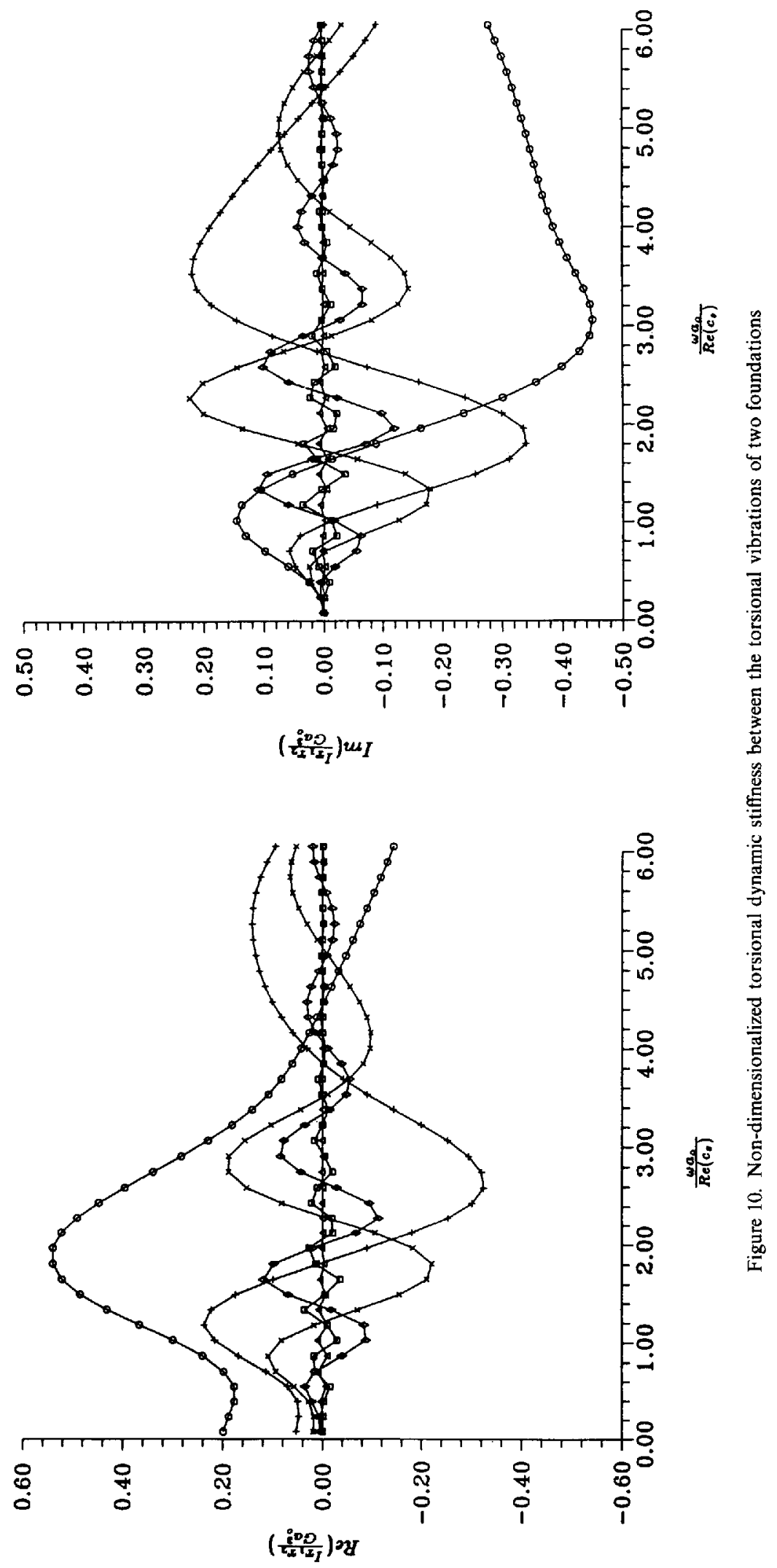
3. The influence on the dynamic stiffness function by the existence of another foundation is not negligible if the two foundations are sufficiently close, (say $d / a_{0} \leqslant 4$ ).

\section{SOME CHARACTERISTICS OF THE PRESENT METHOD}

After invesigation of the present method, several important features were found.

1. Most computational effort in this method is devoted to the calculation of the surface integrals (e.g. $\int_{0}^{a_{1}} \int_{0}^{2 \pi}$ $\left.X X X r_{1} \mathrm{~d} \theta_{1} \mathrm{~d} r_{1}\right)$ in equations 10(a)-13(b) and matrices $\overline{\mathbf{D}}_{n}$ s. However, the calculation of the surface integrals and $\overline{\mathbf{D}}_{n} \mathrm{~s}$ is independent of the complexity of the sub-soil half-space and the excitation frequency, since the changes in the soil properties and excitation frequency are only reflected in matrices $\mathbf{Q}$ and $\overline{\mathbf{Q}}$ in equations 10(a)-13(b). Therefore, one can generate many $\mathbf{K}^{\mathbf{s}}$ and $\mathbf{K}^{\mathrm{a}}$ matrices from equations (22) and (23), corresponding to different excitation frequencies, at the same time. To do this, one just needs to reserve storage space in the computer for these matrices. Actually, this present method needs less computer storage space compared with other methods. This feature would dramatically reduce the computational cost of generating the dynamic stiffness matrices for the interaction of vibrations of several foundations.

2. The method can easily be extended to generating the dynamic stiffness matrices for the interaction of vibrations of more than two foundations.

3. The method can also be extended to calculating the dynamic stiffness matrices for vibrations of foundations with arbitrary shapes. To do this, one needs the co-ordinate transformation matrix for cylindrical- and rectangular co-ordinates. This co-ordinate transformation matrix can be found in Reference 2.

4. Although a half-space medium is assumed throughout the derivation for generating the dynamic stiffness matrix, the present method can be extended to calculating the dynamic stiffness matrices for the case of a layered medium. To do this, one can replace the $\mathbf{Q}$ and $\overline{\mathbf{Q}}$ matrices in equations 10(a) 13(b) with the equivalent matrices for a layered medium. The equivalent matrices can be found in Reference 3.

\section{ACKNOWLEDGEMENTS}

This work was sponsored by National Science Council of Taiwan under contract No. NSC81-0410E009-584. The support is greatly appreciated.

\section{REFERENCES}

1. G.-S. Liou, 'Analytical solution for soil-structure interaction in layered media', Earthquake eng. struct. dyn. 18, 667-686 (1989).

2. G.-S. Liou, 'Vibration of surface foundations for arbitrary shapes', Earthquake eng. struct. dyn. 20, 1115-1125 (1991).

3. G.-S. Liou and G. C. Lee, 'Impedance matrices for axial symmetric foundations on layered media', Structural Eng. Earthquake Eng.. JSCE 9(1), 33-44 (1992).

4. J. Lysmer, 'Vertical motion of rigid footings', Report 3-115, Department of Civil Engineering, University of Michigan, 1965

5. J. E. Luco and R. A. Westmann, 'Dynamic response of circular footings', J. eng. mech. div. ASCE 97, 1381 1395 (1971).

6. H. L. Wong and J. E. Luco, 'Dynamic response of rigid foundations of arbitrary shape', Earthquake eng. struct. dvn. 4. 579 .587 (1976).

7. J. M. Roesset and J. J. Gonzalez, 'Dynamic interaction between adjacent structures', in Proc. DMSR 77, Karlsruhe, Germany, 1977, pp. $127-166$.

8. H.-T. Lin, J. M. Roesset and J. L. Tassoulas, 'Dynamic interaction between adjacent foundations', Earthquake enq. struct. dyn. 15. $323-343$ (1987).

9. G. B. Warburton, J. D. Richardson and J. J. Webster, 'Harmonic response of masses on an elastic half-space'. J. eny. ind. trans. ASME 194, 193-200 (1972)

10. S. A. Savidis and T. Richter, 'Dynamic interaction of rigid foundations', Proc. 9th int. conf'. soil mech. found. eng. Tokyo, 2, 1977. pp. $369-374$

11. J. E. Luco and L. Contesse, 'Dynamic structure-soil-structure interaction', Bull. seism. soc. Am. 63, $1289-1303$ (1973).

12. H. Murakami and J. E. Luco, 'Seismic response of a periodic array of structures', J. eng. mech. div. ASCE 103, $965-977$ (1977).

13. H. L. Wong and J. E. Luco, 'Dynamic interaction between rigid foundations in a layered half-space', Soil dyn. earlhquake eng. 5. $149-158(1986)$

14. H. Kawakami and S. Tasaki, 'Soil-structure interaction of rigid structures considering through-soil coupling between adjacent structures', Proc. 9th world conf. earthquake eng., Tokyo-Kyoto, Japan, Vol. III, 1988, pp. 385-390.

15. Th. Triantafyllidis and B. Prange', Dynamic subsoil-coupling between rigid, circular foundations on the halfspace; Soil $d i n$. earthquake eng. 8, 9-21 (1989).

16. Th. Triantafyllidis and B. Prange', Dynamic subsoil-coupling between rigid rectangular foundations', Soil dyn. earthquake eng. 6. 164-179 (1987). 